\title{
Acessibilidade aos serviços de saúde na Atenção Básica do Estado de Goiás
}

\section{Accessibility to primary health care services in the state of Goiás}

Juliana Pires Ribeiro ${ }^{1}$, Samira Nascimento Mamed ${ }^{2}$, Marta Rovery Souza ${ }^{3}$, Márcia Maria de Souza ${ }^{4}$, Claci Fátima Weirich Rosso ${ }^{5}$

\footnotetext{
${ }^{1}$ Enfermeira, Mestre em Enfermagem. Enfermeira da Secretaria Municipal de Saúde de Goiânia e da Secretaria Municipal de Saúde de Aparecida de Goiânia. Goiânia, GO, Brasil. E-mail: julianapribeiro@yahoo.com.br.

${ }^{2}$ Enfermeira. Discente do Programa de Pós-Graduação em Enfermagem, nível Mestrado, da Faculdade de Enfermagem (FEN) da Universidade Federal de Goiás (UFG). Enfermeira da Secretaria Municipal de Saúde de Goiânia. Goiânia, GO, Brasil. E-mail: samiramamed31@gmail.com.

${ }^{3}$ Bacharel em Ciências Sociais, Doutora em Ciências Sociais. Professora Associado do Instituto de Patologia Tropical e Saúde Publica da UFG. Goiânia, GO, Brasil. E-mail: martary@gmail.com.

${ }^{4}$ Enfermeira, Doutora em Ciências da Saúde. Professora Adjunto da FEN/UFG. Goiânia, GO, Brasil. E-mail: marcia@fen.ufg.br.

${ }^{5}$ Enfermeira, Doutora em Ciências da Saúde. Professora Adjunto da FEN/UFG. Goiânia, GO, Brasil. E-mail: claci.proec.ufg@gmail.com.
}

\section{RESUMO}

Objetivou-se analisar a acessibilidade aos serviços de saúde na Atenção Básica no estado de Goiás. Estudo descritivo de corte transversal com base nos dados secundários proveniente do Programa Nacional de Melhoria do Acesso e da Qualidade da Atenção Básica. A amostra do estudo foi constituída por profissionais de saúde das 1.216 UBS. Verificouse que, a sala de acolhimento está ausente em $68,5 \%$ das unidades de saúde, o que prejudica, consideravelmente, os profissionais na tomada de decisão imediata. A ausência de consultórios clínicos em 2\% dos locais dificulta a acessibilidade aos serviços de saúde na Atenção Básica em Goiás. Com relação ao horário e turno de funcionamento, verificou-se que $86 \%$ das unidades atendem cinco dias na semana em turnos de oito horas, não favorecendo a acessibilidade do usuário. Com este estudo constata-se a deficiência da acessibilidade aos serviços de saúde e a necessidade de maiores investimentos no fortalecimento da Atenção Básica.

Descritores: Acessibilidade aos Serviços de Saúde; Atenção Primária à Saúde; Avaliação em Saúde; Enfermagem em Saúde Comunitária.

\section{ABSTRACT}

The objective of this study was to evaluate accessibility to primary health care services in the state of Goiás. A descriptive cross-sectional study was conducted based on secondary data from the National Program to Improve Access to and Quality of Primary Health Care. The study sample was composed of health professionals from 1,216 primary health care units. Results showed that $68.5 \%$ of the health units miss a screening room, thus considerably damaging prompt decision-making by professionals. The lack of medical offices in $2 \%$ of the sites hinders the primary health care services accessibility in Goiás. As regards opening hours and work shifts, $86 \%$ of the units are open five days a week in eighthour shifts, which does not favor accessibility for users. This study confirms the lack of accessibility to health services and the need for additional investments to strengthen primary health care.

Descriptors: Health Services Accessibility; Primary Health Care; Health Evaluation; Community Health Nursing. 


\section{INTRODUÇÃO}

A acessibilidade aos serviços de saúde na Atenção Básica tem sido relatada na literatura como um dos principais problemas relacionados à assistência ${ }^{(1-2)}$. Esses problemas possuem ligação com a qualidade dos serviços, gestão ineficaz, dificuldade para a marcação de consultas no mesmo dia da procura pelo atendimento, filas para realização do agendamento, longo tempo de espera e distância das unidades em algumas regiões ${ }^{(3)}$.

A acessibilidade aos serviços de saúde na Atenção Básica possui relação direta com a consolidação dos princípios do Sistema Único de Saúde - SUS e pode ser definida como uma das dimensões do acesso(4). Dessa forma, diz respeito à resolutividade dos serviços de modo que a oferta seja suficiente às necessidades da população no momento da procura pelo atendimento ${ }^{(5)}$.

O conceito de acesso e acessibilidade são empregados de diferentes formas e podem sofrer variações conforme o contexto e no decorrer do tempo. $\mathrm{Na}$ literatura encontramos autores que utilizam o termo acesso e acessibilidade e seus sinônimos, e outros que fazem distinção de seus significados ${ }^{(1,4,6)}$.

O conceito de acesso aos serviços de saúde é considerado como a liberdade de escolher os serviços e sua disponibilidade no momento da procura pelo atendimento $^{(2,4,6)}$. Ele também pode ser entendido como a associação entre alguns elementos denominados disponibilidade, aceitabilidade e informação e cada vez mais se confunde com o conceito de equidade em saúde $^{(7)}$.

A acessibilidade pode também ser considerada como uma das dimensões do acesso. Dessa forma, diz respeito a relação entre as características do Sistema de Saúde e da população que ele serve, ou ainda, ao grau de ajuste entre as características da população e da oferta de serviços $^{(4)}$.

Quando se fala em acessibilidade, logo se questiona a qualidade dos serviços e a forma como eles estão sendo oferecidos à população. Segundo alguns autores, para estimar a qualidade dos serviços de saúde, torna-se essencial a avaliação da estrutura, do processo e do resultado $^{(8)}$.

O planejamento adequado dos serviços de saúde, considerando a estrutura física disponível na Atenção Básica, contribui para o processo de trabalho mais adequado, proporcionando maior qualidade e acessibilidade para os usuários que buscam atendimento nos estabelecimentos de saúde.

A qualidade dos serviços de saúde na Atenção Básica vem sendo discutida desde a década de $30^{(9)}$. Já a avaliação dos serviços de saúde direcionada para a qualidade da assistência conquistou espaço no Brasil no final da década de 1990 com o fortalecimento do controle social e a valorização da participação da comunidade nas ações de planejamento e avaliação ${ }^{(10)}$.

$\mathrm{Na}$ busca pela qualidade dos serviços, os processos avaliativos estão diretamente relacionados com o desenvolvimento da gestão, o que vem envolvendo os profissionais da saúde que, tradicionalmente, não possuem a cultura de avaliação e monitoramento. Para construção dessa cultura, há necessidade de fortalecer parcerias entre o ensino, serviço e gestão, no sentido de dar sustentação à formação gerencial dos profissionais numa perspectiva transformadora ${ }^{(11)}$.

No âmbito do desenvolvimento da gestão, para melhorar a qualidade dos serviços de saúde, é necessário estruturar as Unidades Básicas de Saúde - UBS, melhorando alguns problemas que se refletem na acessibilidade ineficaz de alguns serviços, na insuficiência e no perfil inadequado dos gestores e trabalhadores, no difícil acesso aos insumos, na pouca efetividade de políticas e ações de saúde e na inadequada estrutura física de algumas unidades de saúde ${ }^{(12)}$.

Devido aos problemas citados acima, o Ministério da Saúde - MS almeja executar a gestão pública com base na indução, monitoramento e avaliação de processos e resultados mensuráveis, garantindo acessibilidade e qualidade da atenção em saúde para toda a população ${ }^{(13)}$.

Nesse sentido, foram propostas várias iniciativas centradas na qualificação da Atenção Básica. Dentre elas, 
destaca-se o Programa Nacional de Melhoria do Acesso e da Qualidade da Atenção Básica - PMAQ - $A B^{(13)}$.

Esse programa foi instituído pela portaria de $\mathrm{n} 01.654$ GM/MS, do dia 19 de julho de 2011 e foi produto de um importante processo de negociação e pactuação das três esferas de gestão do SUS $^{(13)}$. Ele é desenvolvido em quatro fases que se complementam e que configuram um ciclo contínuo da melhoria do acesso e da qualidade da Atenção Básica e são, assim, denominadas: Adesão e Contratualização; Desenvolvimento; Avaliação Externa e Recontratualização ${ }^{(14)}$.

O PMAQ, além de estimular que as equipes de Atenção Básica se autoavaliem e ofertem serviços que assegurem maior acesso e qualidade, de acordo com as necessidades concretas da população, tornou-se um desafio para os gestores. Esse programa também busca garantir um padrão de qualidade comparável nacional, regional e localmente, de maneira a permitir maior transparência e efetividade das ações governamentais direcionadas à Atenção Básica em todo o Brasil ${ }^{(15)}$.

Os desafios da gestão na Atenção Básica dos serviços públicos de saúde são inúmeros e têm sido alvo de discussões nesses últimos anos, especialmente com a gestão da saúde descentralizada aos municípios, conforme prevê o SUS. Uma das dificuldades que permeiam essas discussões é a gestão de recursos humanos e materiais nos serviços de saúde. A produção dos serviços de saúde depende não só de recursos materiais, tecnológicos e de uma estrutura física adequada, como também de profissionais, devidamente, qualificados para transformar insumos em resultados ${ }^{(16)}$.

Com o desenvolvimento da gestão e a necessidade da realização de processos avaliativos, fez-se necessária a criação de instrumentos que abordassem essa temática. Desta forma, a presente pesquisa teve como objetivo analisar a acessibilidade aos serviços de saúde na Atenção Básica do estado de Goiás por meio de uma pesquisa nacional de avaliação da Atenção Básica realizada pelo Ministério da Saúde.

\section{MÉTOdOS}

Estudo descritivo de corte transversal que tem como fonte uma base de dados secundários proveniente do primeiro ciclo do componente de avaliação externa do Programa Nacional de Melhoria do Acesso e da Qualidade da Atenção Básica - PMAQ - AB em Goiás, cuja coleta foi realizada entre os meses de julho a setembro de 2012, pelo Departamento de Atenção Básica - DBA do Ministério da Saúde em parceria com instituições de pesquisa e ensino superior.

O instrumento de coleta de dados foi constituído por três módulos. O primeiro abordou aspectos relacionados as condições de infraestrutura, o segundo caracterizou a organização dos serviços e o processo de trabalho por meio de entrevista com os profissionais de saúde e o terceiro diz respeito à entrevista com o usuário no intuito de analisar sua satisfação e percepção quanto aos serviços de saúde no que se refere ao seu acesso e utilização. No presente estudo foram utilizados dados oriundos do primeiro módulo, relacionados as condições de infraestrutura que estão vinculados a acessibilidade aos serviços de saúde.

Como logística do processo de avaliação externa da Atenção Básica no Brasil/MS, os estados brasileiros foram agrupados em cinco consórcios. O estado de Goiás faz parte do consórcio composto pelos estados do Rio Grande do Sul, Santa Catarina, Minas Gerais, Maranhão e Distrito Federal, cuja coordenação geral está localizada na Universidade Federal de Pelotas - RS.

O PMAQ foi desenvolvido em quatro fases: a primeira corresponde à adesão e contratualização por parte dos municípios; a segunda constitui-se no desenvolvimento, autoavaliação, monitoramento, educação permanente e apoio institucional; a terceira é composta pela avaliação externa e a quarta é formada pela recontratualização.

A amostra do estudo foi constituída por profissionais de saúde das 1216 UBS dos 246 municípios do estado de Goiás que responderam ao módulo I da terceira fase da pesquisa que consiste na avaliação externa para a totalidade de municípios e Equipes da Atenção Básica - 
$E A B$, participantes do Programa. Como critério de exclusão, foi utilizada a ausência dos profissionais na equipe por motivo de férias ou licenças.

Os dados foram coletados no âmbito do PMAQ-AB (primeiro ciclo), no componente de Avaliação Externa, no qual ocorreu a aplicação de três módulos de questionários em meio eletrônico. A coleta de dados foi realizada por oito equipes em diferentes rotas. Para essa divisão foi utilizado mapas das rodovias e dos rios que cortam o estado de Goiás, pela associação das divisões de micro e macrorregiões existentes e pelo apoio da Superintendência de Políticas de Atenção Integral à Saúde - SPAIS da Secretaria Estadual de Saúde de Goiás.

Cada equipe foi composta por uma supervisora de campo com uma média de quatro entrevistadores, devidamente treinados para realização da pesquisa.

Para a coleta de dados, foi utilizado um instrumento estruturado, padronizado e previamente validado para a avaliação externa das UBS. Esse instrumento foi estruturado por uma equipe do MS.

O estudo foi aprovado pelo Comitê de Ética em Pesquisa da Universidade Federal de Pelotas com o número de protocolo 38/2012 e teve como base os cuidados éticos necessários para pesquisa com seres humanos preconizados pela Resolução 466/2012, da Comissão Nacional de Ética em Pesquisa - CONEP.

Ao final de cada entrevista, os dados foram enviados para um banco do MS, por meio da utilização de tablets e via uma rede de internet. Após o envio, esses dados foram armazenados e agrupados em módulos I, II e III. A análise estatística foi realizada por meio do Software Statistical Package of Social Sciences - SPSS versão 19.0.

\section{RESULTADOS}

Nesse estudo, trabalhou-se com profissionais de saúde das 1.216 UBS que integram a Atenção Básica do estado de Goiás. Essas unidades foram incluídas no primeiro ciclo da pesquisa nacional de avaliação realizada pelo Ministério da Saúde, por meio do Programa Nacional de Melhoria do Acesso e da Qualidade da Atenção Básica - PMAQ-AB, respondendo ao módulo I da pesquisa.

Por meio da análise dos dados, foi possível construir duas categorias: ambientes que melhoram a acessibilidade e condições do ambiente que favorecem a acessibilidade.

\section{Ambientes que melhoram a acessibilidade}

Os ambientes que melhoram a acessibilidade estão relacionados a espaços que proporcionam informações, privacidade, humanização, resolutividade, oferta e disponibilização de serviços. Esses ambientes são importantes na consolidação da acessibilidade, pois eles proporcionam aos usuários maior proximidade com o serviço.

Nos ambientes que compõem a estrutura física das unidades de saúde na Atenção Básica, destaca-se que a maioria delas conta com recepção e sala de espera conforme pode ser observado na Figura 1. Situação contrária acontece com a sala de acolhimento, pois 834 $(68,5 \%)$ unidades não possuem esse ambiente.

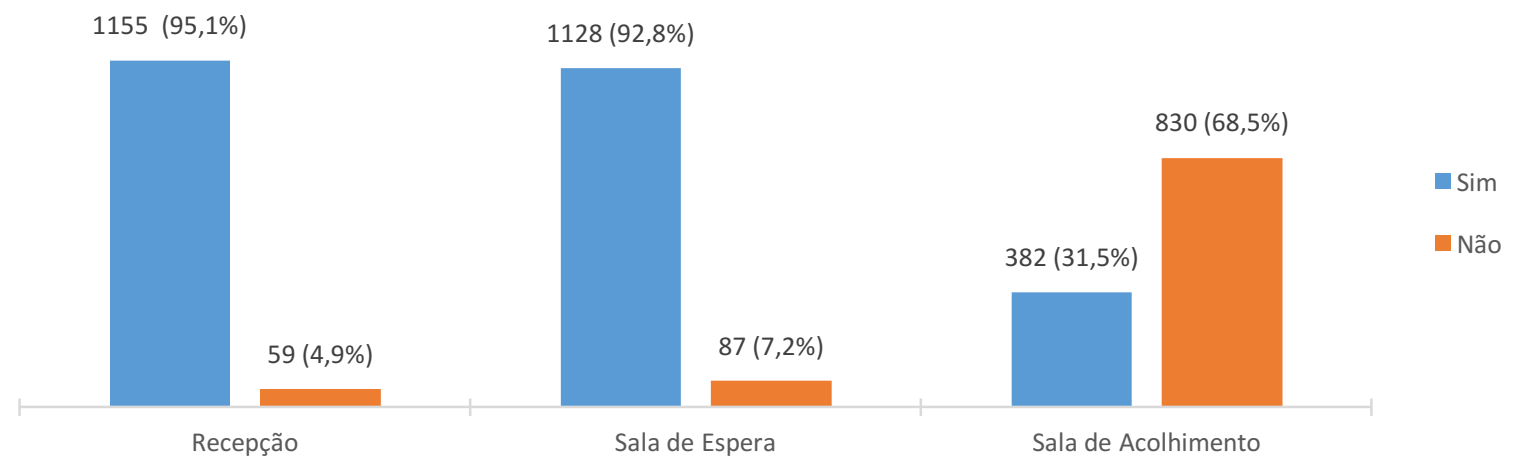

Figura 1: Ambientes que compõem a estrutura física das unidades de saúde na Atenção Básica do estado de Goiás, 2012. Fonte: Dados do PMAQ-AB - Goiás - primeiro ciclo, 2012. 
Em relação aos ambientes destinados à farmácia e à sala de estocagem de medicamentos, o resultado mais evidente observado, nas unidades de saúde, foi a presença de uma farmácia por estabelecimento, porém destaca-se ainda, na Figura 2, a ausência de sala de estocagem de medicamentos em 956 (79\%) unidades de saúde, o que pode dificultar na resolutividade do tratamento proposto pelos profissionais presentes nas unidades.

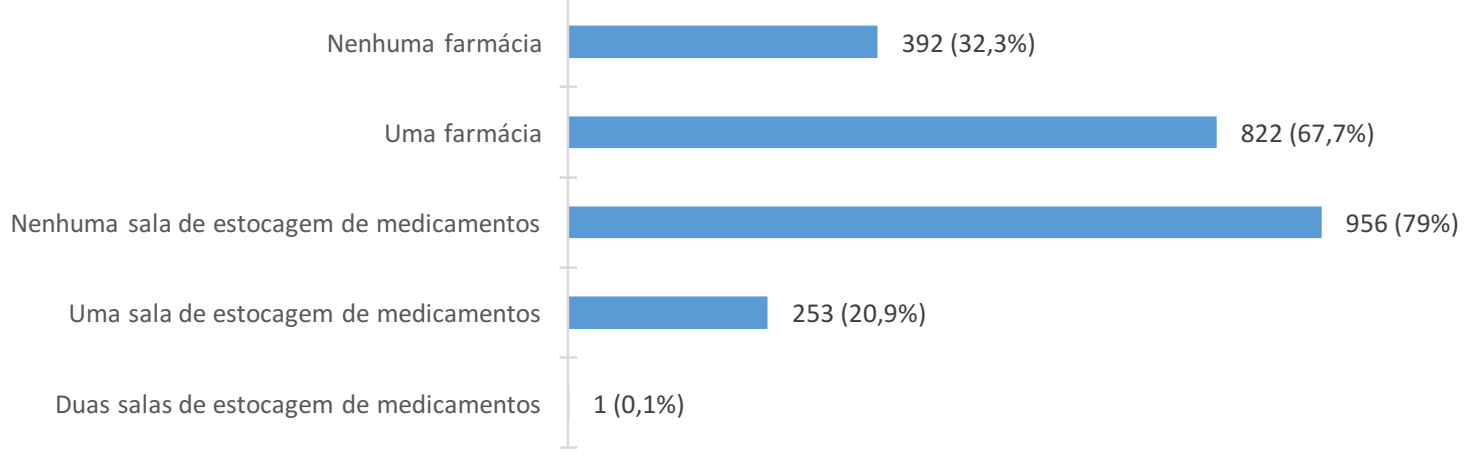

Figura 2: Quantidade de farmácias e salas de estocagem de medicamentos das unidades de saúde na Atenção Básica do estado de Goiás, 2012.

Fonte: Dados do PMAQ-AB - Goiás - primeiro ciclo, 2012.

Outros ambientes importantes para melhorar a acessibilidade são descritos pelo número de consultórios clínicos e odontológicos. A quantidade de um a três consultórios clínicos está presente em 1.064 (88\%) unidades, mais de quatro em 123 (10\%) e ausência de consultórios em 29 (2\%) UBS.

Com relação ao número de consultórios odontológicos, foi encontrado de um a três consultórios em 920 (76\%) unidades, mais de quatro, em nove $(0,7 \%)$ locais e a ausência desses ambientes em 287 (23,3\%) estabelecimentos de saúde.

Esse resultado retrata a realidade vivenciada pelos serviços de saúde na Atenção Básica do estado de Goiás na qual, em algumas situações, não é oferecido o serviço de saúde bucal e em outras, quando ele está disponível, a privacidade e o sigilo não são garantidos, pois os ambientes não são individualizados e atendem simultaneamente três usuários no mesmo ambiente.

Nas salas de procedimentos destinadas ao atendimento do usuário, descritas na Tabela 1, é possível identificar que 852 (70\%) unidades de saúde dispõem de salas de curativo, 592 (48,6\%), de salas de procedimentos e $893(73,4 \%)$, de salas de vacina. A ausência de alguns ambientes na maior parte das unidades como salas de nebulização, salas de reunião, salas de esterilização e guarda e salas de observação prejudicam o atendimento, levando a reclamações e situações que poderiam ser evitadas com o planejamento adequado dos serviços.

Tabela 1: Salas de procedimentos das unidades de saúde na Atenção Básica do estado de Goiás, 2012.

\begin{tabular}{|c|c|c|c|c|}
\hline Variável & Frequência $(n=1216)$ & $\%$ & Frequência $(n=1216)$ & $\%$ \\
\hline Salas diversas & Sim & & Não & \\
\hline Sala de Curativo & 852 & $70,0 \%$ & 364 & $30,0 \%$ \\
\hline Sala de Procedimento & 592 & $48,6 \%$ & 624 & $51,4 \%$ \\
\hline Sala de nebulização & 440 & $36,1 \%$ & 776 & $63,9 \%$ \\
\hline Sala de vacina & 893 & $73,4 \%$ & 323 & $26,6 \%$ \\
\hline Sala de esterilização e guarda & 500 & $41,2 \%$ & 716 & $58,8 \%$ \\
\hline Sala de observação & 261 & $21,5 \%$ & 955 & $78,5 \%$ \\
\hline Sala de reuniões & 404 & $33,3 \%$ & 812 & $66,7 \%$ \\
\hline
\end{tabular}

Fonte: Dados do PMAQ-AB - Goiás - primeiro ciclo, 2012. 
Os ambientes demonstrados nas Figuras 1, 2 e Tabela 1 acima estão diretamente relacionados ao atendimento ao público, enquanto que outros ambientes dão suporte a esse atendimento como, por exemplo, a existência de sanitários masculinos, femininos e para deficientes físicos que são fatores importantes na acessibilidade aos serviços de saúde.
Foi possível observar que as unidades de saúde apresentam uma maior quantidade de banheiros individualizados masculinos do que femininos. Outra informação relevante e que dificulta a acessibilidade está na ausência de banheiros adaptados para pessoas com deficiência em 1.032 (85\%) ambientes (Tabela 2).

Tabela 2: Informações sobre sanitários das unidades de saúde na Atenção Básica do estado de Goiás, 2012.

\begin{tabular}{|c|c|c|}
\hline Variável & Frequência $(n=1216)$ & $\%$ \\
\hline \multicolumn{3}{|l|}{ Sanitário masculino para usuário } \\
\hline Não & 296 & 24,3 \\
\hline Sim & 920 & 75,7 \\
\hline \multicolumn{3}{|l|}{ Sanitário feminino para usuários } \\
\hline Não & 364 & 30,0 \\
\hline Sim & 852 & 70,0 \\
\hline \multicolumn{3}{|l|}{ Sanitário adaptado para pessoas com deficiência } \\
\hline Não & 1034 & 85,0 \\
\hline Sim & 182 & 15,0 \\
\hline
\end{tabular}

Ainda dentro dessa abordagem dos serviços de apoio, o espaço de acondicionamento do lixo contaminado gerado nas unidades de saúde deve ter um local adequado para não se tornar um foco de contaminação. Entretanto, a maioria dos estabelecimentos de saúde 749 (61,5\%) não conta com esse local e, apenas, 467 (38,5\%) possuem esse ambiente.

\section{Condições do ambiente que favorecem a acessibilidade}

As condições do ambiente que favorecem a acessibilidade são descritas com relação à presença de ampliação ou reforma dos serviços de saúde, sinalização interna ou externa das unidades, turnos de atendimento, presença de calçada em boas condições, tapetes, corrimão, entre outros.

A presença dessas condições proporciona aos usuários um atendimento equitativo e mais resolutivo. Das 1.216 UBS, 60 (4,9\%) estavam em processo de ampliação e 85 (7\%), em processo de reforma. No que se refere ao processo de ampliação para melhorar a acessibilidade é importante ressaltar que 27 (2,2\%) UBS estavam ampliando banheiros adaptados para pessoas com deficiência física.
Algumas ações relacionadas aos processos de reforma (85 UBS) interferem na acessibilidade, dentre elas pode-se destacar a reforma nos pisos em 55 (64,7\%) unidades, nas instalações elétricas em 52 (61,2\%) serviços de saúde e nas instalações hidrossanitárias em 51 (60\%) locais estudados.

$A$ acessibilidade aos serviços de saúde e as condições do ambiente que a favorecem, também possuem relação com os dias da semana que a unidade funciona, com os turnos de atendimento e com o horário de funcionamento, pois interferem na disponibilidade do usuário em poder procurar esses serviços.

Sendo assim, observa-se que 1.046 (86\%) unidades atendem cinco dias na semana e 170 (14\%) estabelecimentos atendem mais de cinco dias. Em relação aos turnos de atendimento, identifica-se que 98 (8\%) unidades realizam atendimento em um turno do dia, 1.070 (88\%) delas em dois turnos e 48 (4\%) locais atendem em três turnos.

Com relação ao horário de funcionamento, percebese que algumas unidades encerram o atendimento até às $13 \mathrm{~h}$, outras entre às $14 \mathrm{~h}$ e às $18 \mathrm{~h}$ e uma pequena parte 
funciona até às $19 \mathrm{~h}$. Por fim, destaca-se que 40,2\% das unidades funcionam durante o horário de almoço.

Ainda no que se refere às condições que favorecem a acessibilidade as sinalizações externa e interna são informações relevantes quando possuem a intenção de nortear o usuário no serviço, diminuindo as reclamações e as situações conflituosas. Dessa forma, destaca-se, na sinalização externa das unidades de saúde, a ausência de totem externo em 1.100 (90,4\%) unidades, 809 (66,5\%) delas possuem faixa como sinalização, mas não estão adequadas para a identificação da unidade de saúde e 575 (47,3\%) utilizam outras formas de sinalização.

Nas informações divulgadas para os usuários na parte interna da UBS, destacam-se a presença do horário de funcionamento em 610 (50,2\%) e a ausência de uma lista com a descrição dos serviços oferecidos em 678 (55,8\%) unidades de saúde.

Com relação às condições que favorecem a acessibilidade para os deficientes auditivos, visuais ou pessoas não alfabetizadas, percebe-se a ausência de símbolos internacionais em 1.209 (99,4\%) ambientes; de figuras, desenhos ou cores em 1.124 (92,4\%) serviços; de caracteres em relevo em 1.214 (99,8\%) locais; de recursos auditivos em 1.214 (99,8\%) estabelecimentos de saúde e de profissionais para acolhimento em 1.215 (99,9\%) unidades.

Ainda no que se refere aos portadores de deficiência física, é possível observar as dificuldades relacionadas à acessibilidade nos serviços de saúde, quando se destacam as condições da calçada, tapete, piso, rampa, corrimão, portas e corredores (Tabela 3).

Tabela 3: Acessibilidade das unidades de saúde da Atenção Básica no estado de Goiás para deficientes físicos, 2012.

\begin{tabular}{|c|c|c|}
\hline Variável & Frequência $(n=1216)$ & $\%$ \\
\hline \multicolumn{3}{|l|}{ Calçada em boas condições } \\
\hline $\operatorname{sim}$ & 479 & 39,4 \\
\hline Não & 737 & 60,6 \\
\hline \multicolumn{3}{|l|}{ Possui tapete } \\
\hline Sim & 234 & 19,2 \\
\hline Não & 982 & 80,8 \\
\hline \multicolumn{3}{|l|}{ Piso antiderrapante } \\
\hline $\operatorname{Sim}$ & 280 & 23,0 \\
\hline Não & 936 & 77,0 \\
\hline \multicolumn{3}{|l|}{ Piso regular } \\
\hline Sim & 640 & 52,6 \\
\hline Não & 576 & 47,4 \\
\hline \multicolumn{3}{|l|}{ Rampa } \\
\hline Sim & 531 & 43,7 \\
\hline Não & 685 & 56,3 \\
\hline \multicolumn{3}{|l|}{ Corrimão } \\
\hline Sim & 68 & 5,6 \\
\hline Não & 1148 & 94,4 \\
\hline \multicolumn{3}{|l|}{ Porta e corredores adaptados } \\
\hline Sim & 375 & 30,8 \\
\hline Não & 841 & 69,2 \\
\hline
\end{tabular}

A maioria dos dados encontrados reflete a ausência de acessibilidades para os portadores de deficiência física, exceto quando observado sobre a presença de piso regular que, na maior parte das unidades, 640 (52,6\%) constaram-se oferecer boas condições.

\section{DISCUSSÃO}

Para analisar as condições de acessibilidade, foram adotadas duas categorias. Na primeira, destacam-se os ambientes que melhoram a acessibilidade e dentre eles a sala de acolhimento que está ausente em $68,5 \%$ das unidades, o que prejudica os profissionais na tomada de 
decisão imediata. A inexistência desse ambiente nas unidades de saúde prejudica o atendimento, na medida em que os profissionais passam a ficar desprovidos de um local adequado para priorizar o atendimento conforme a necessidade individual e prioritária de cada usuário, acarretando demora ou atendimento inadequado.

Nesse sentido, a Política Nacional de Humanização traz como definição de acolhimento a "capacidade de acolhida das demandas e necessidades como pressuposto básico do contrato entre unidade de saúde e usuário e sua rede sociofamiliar". Essa mesma política, ainda aposta que o acolhimento é uma das maneiras de organizar os processos de trabalho em saúde, o que facilita no enfrentamento dos problemas e potencializa a criação de alternativas criativas e individualizadas em cada contexto $^{(17)}$.

Ainda com relação aos ambientes que favorecem a acessibilidade, a maioria (79\%) das unidades não conta com sala específica para estocagem de medicamentos e, além disso, outras unidades de saúde não possuem farmácia. Esses locais são importantes na perspectiva da assistência farmacêutica, uma vez que prover a medicação significa dar continuidade ao tratamento estabelecido pelos profissionais de saúde e reafirma o princípio da integralidade estabelecido na Constituição Federal de 1988. Na ausência desses ambientes e com a falta de condição financeira da maioria dos usuários, o tratamento não consegue ser resolutivo, o que reflete negativamente nos serviços de saúde da Atenção Básica $^{(18)}$.

A infraestrutura adequada para armazenamento e distribuição de medicamentos contribui para a diminuição dos erros relacionados à dispensação dessas drogas e propicia o desenvolvimento correto da terapêutica prescrita pelos profissionais ${ }^{(19)}$.

Dessa forma, a farmácia possui a função de dar continuidade na assistência e garantir a qualidade desse serviço por meio do uso seguro e racional de medicamentos, adequando sua aplicação à saúde individual e coletiva na assistência à saúde e prevenção à doença $^{(18)}$.

O manual de estrutura física das UBS preconiza que os consultórios clínicos devem ter no mínimo uma área de $9 \mathrm{~m}^{2}$, lavatório com torneiras de fechamento que dispense o uso das mãos, porta-papel toalha, porta-dispensador de sabão líquido, uma mesa tipo escritório com gavetas, três cadeiras, mesa de exame clínico ou mesa para exame ginecológico, uma banqueta giratória cromada, uma escada com dois degraus, um biombo duplo, um foco com haste flexível, um armário vitrine, lixeira com tampa e pedal, telefone e computador ${ }^{(20)}$.

Nas 1.216 UBS pesquisadas, $2 \%$ dos locais não possuíam consultórios clínicos e nas unidades de saúde com consultórios, esses ambientes não tinham condições mínimas conforme preconizado pelo MS para serem considerados como consultórios clínicos. A ausência da adequação desses ambientes denota que essas unidades precisam de maiores investimentos, a fim de oferecer um serviço resolutivo, equitativo e que forneça privacidade ao usuário.

Em um estudo ${ }^{(21)}$ realizado nas Unidades Básicas de Saúde da Família - UBSF em Fortaleza, percebeu-se que todas as unidades possuíam características semelhantes a essa pesquisa com consultórios em quantidades reduzidas para o número de profissionais e equipamentos não condizentes com uma estrutura ergonômica.

Nas salas de procedimentos estudadas, foi possível perceber que algumas unidades de saúde compartilham do mesmo ambiente para a realização de alguns procedimentos como nebulização, sala de curativo, sala de esterilização e guarda, e sala de observação. Essa situação pode prejudicar o atendimento ao usuário, na medida em que aumenta consideravelmente o risco de infecção, pois não possuem separação do que é limpo e contaminado.

Ainda no que se refere às estruturas ou espaços essenciais existentes nas unidades de saúde para o atendimento das necessidades da população com qualidade, destaca-se que muitos ambientes não contam 
com expurgo para a limpeza de instrumentos utilizados em diversos procedimentos como curativos, o que denota que essas unidades de saúde não estão dentro dos padrões preconizados pelo $\mathrm{MS}^{(20)}$.

A ausência de sanitários para portadores de deficiência em $85 \%$ das unidades de saúde mostra a necessidade de mudanças para que seja ofertada a esses usuários, ambientes que proporcionem acessibilidade digna, com bem estar e satisfação.

Em um estudo ${ }^{(22)}$ realizado em sete estados do Brasil, constatou-se que $77,4 \%$ dos banheiros das UBS não existiam portas que garantissem o acesso de cadeirantes e em $75,8 \%$ dos banheiros não era possível realizar manobras de aproximação com a cadeira de rodas. Esse resultado corrobora a pesquisa realizada nas UBS do estado de Goiás, mostrando a necessidade de investimentos na inclusão social de todos os usuários que buscam por atendimento.

Dados de outra pesquisa realizada em Pernambuco ${ }^{(1)}$ reafirmam a importância dos achados encontrados no estado de Goiás, onde encontrou-se a inexistência de banheiros adaptados em $97 \%$ das UBS visitadas.

Dentro das condições que favorecem a acessibilidade está a sinalização interna e externa das UBS. No presente estudo foi possível observar que a maior parte das unidades não possui essas sinalizações de forma adequada, dificultando a comunicação dos usuários com o serviço.

Outro estudo ${ }^{(23)}$ realizado em seis UBS de Fortaleza, aponta que os usuários são os que mais sofrem com a falta de comunicação, pois nesses locais a sinalização e a comunicação funcionam de forma muito precária e muitas vezes isolada, dificultando as relações entre os trabalhadores e entre esses e os usuários.

As UBS do estado de Goiás necessitam rever a sinalização quanto à acessibilidade aos deficientes auditivos, visuais ou pessoas não alfabetizadas. $\mathrm{Na}$ utilização de símbolos internacionais, apenas, sete unidades de saúde em todo o estado de Goiás possuem esse tipo de sinalização. Caracteres em relevo e recursos auditivos foram encontrados somente em duas UBS.

No que se refere à acessibilidade das unidades de saúde para deficientes físicos, idosos e demais usuários, os pisos devem ter superfície regular, firme, estável e antiderrapante sob qualquer condição e não devem provocar trepidação em dispositivos com rodas ${ }^{(20)}$. Nas UBS do estado de Goiás, 52,6\% possuem piso regular, porém a presença de piso antiderrapante e calçadas em boas condições não foram observadas com a mesma frequência, ou seja, grande parte dos estabelecimentos de saúde não está adequada, conforme preconiza o MS; apresentando, portanto, barreiras arquitetônicas, o que proporciona um aumento do risco de acidentes e dificulta consideravelmente a acessibilidade das pessoas.

Em um estudo ${ }^{(24)}$ realizado no Rio Grande do Norte, verificou-se que a acessibilidade das pessoas com deficiência nos mais diversos ambientes é uma garantia de extrema relevância a ser conquistada. Nas unidades de saúde da Atenção Básica do estado de Goiás, essa conquista também deve ser alvo de desejo dos gestores e da comunidade, pois segundo a Política Nacional de Saúde da Pessoa com Deficiência, os estados e os municípios devem garantir o princípio da integralidade e as ações destinadas a atender às necessidades dessa população referente à saúde, educação, trabalho, relacionamento afetivo e social.

A ideia de integralidade preconizada pelo SUS gera a expectativa que os gestores e profissionais de saúde reconheçam os usuários como sujeitos de fato, de direito, e com necessidades básicas que precisam ser atendidas. Entretanto, a inacessibilidade das pessoas nas UBS compromete a proposição da integralidade como componente fundamental de cuidado em saúde e, consequentemente, compromete, também, a produção de sujeitos ${ }^{(25)}$.

\section{CONCLUSÃO}

A acessibilidade dos usuários aos serviços de saúde na Atenção Básica despertou o interesse do Ministério da 
Saúde, dada a importância dessa abordagem na avaliação da qualidade dos serviços. Ao investigar como ocorre a acessibilidade dos usuários na Atenção Básica à Saúde, a partir do estudo de avaliação externa das UBS do estado de Goiás participantes do Programa Nacional de Melhoria do Acesso e da Qualidade da Atenção Básica, foi possível apontar quais são os fatores que dificultam a acessibilidade nos serviços básicos de saúde.

Em relação aos ambientes que melhoram a acessibilidade, pode-se afirmar que em grande parte das UBS, não existem sala de acolhimento, sala de estocagem de medicamentos, salas de procedimentos e sanitários para portadores de deficiência, dificultando, consideravelmente, a acessibilidade aos serviços de saúde.

Ao analisar a sinalização interna das UBS, pisos antiderrapantes, rampas, corrimão, calçadas em boas condições, portas e corredores adaptados, pode-se

\section{REFERÊNCIAS}

1. Albuquerque MSV, Lyra TM, Farias SF, Mendes MFM, Martelli PJL. Acessibilidade aos serviços de saúde: uma análise a partir da Atenção Básica em Pernambuco. Rev Saúde em Debate. 2014; 38(esp): 252-264.

2. Campos RTO, Ferrer AL, Gama CAP, Campos GWS, Trapé TL, Dantas DV. Avaliação da qualidade do acesso na atenção primária de uma grande cidade brasileira na perspectiva dos usuários. Rev Saúde em Debate. 2014; 38(esp): 252-264. 3. Kopach R, De Laurentis P-C, Lawley M, Muthuraman K, Ozsen L, Rardin R, et al. Effects of clinical characteristics on successful open access scheduling. Health Care Manag Sci. 2007; (10):111-24.

4. Travassos C, Martins M. Uma revisão sobre os conceitos de acesso e utilização de serviços de saúde. Cad Saude Publica 2004; 20(Suppl. 2):190-198.

5. Donabedian A. An introduction to quality assurance in health care. New York: Oxford University, 2003.

6. Thiede M, Mclntyre D. Information, communication and equitable access to health care: a conceptual note. Cad Saude Publica 2008; 24(5): 1168-1173.

7. Sanchez RM, Ciconelli RM. Conceitos de acesso à saúde. Rev Panam Salud Publica. 2012; 31(3): 260-268.

8. Morais AS, Melleiro MM. A qualidade da assistência de enfermagem em uma unidade de emergência: a percepção do usuário. Rev. Eletr. Enf. [Internet]. 2013 [acesso em: 09 abr 2014]; 15(1):112-20. Disponível em: http://dx.doi.org/10.5216/ree.v15i1.15243. afirmar que a maioria das unidades de saúde não está dentro dos padrões preconizados pelo MS.

Sendo assim, os resultados desse estudo permitem verificar que a inacessibilidade das unidades de saúde na Atenção Básica do estado de Goiás fere o princípio da integralidade, dificultando a assistência e prejudicando aqueles que precisam dos serviços de assistência à saúde.

Desse modo, essa análise, na perspectiva da gestão visa direcionar ou redirecionar as prioridades governamentais, permitindo o correto investimento no fortalecimento da Atenção Básica.

Outros estudos deverão ser realizados para avaliar a acessibilidade da Atenção Básica à Saúde no estado de Goiás para verificar as melhorias implementadas após os investimentos disponibilizados aos municípios pelo Ministério da Saúde com base nos resultados do primeiro ciclo do PMAQ-AB, a fim de melhorar os ambientes e condições que favorecem a acessibilidade.

9. Lee RI, Jones LW. The fundamentals of good medical care. Chicago, The University of Chicago Press; 1933.

10. Esperidião M, Trad LAB. Avaliação de satisfação de usuários. Ciência \& Saúde Coletiva. 2005; (10): 303-12. 11. Weirich CF, Munari DB, Mishima SM, Bezerra ALQ. O trabalho Gerencial do Enfermeiro na Rede Básica de Saúde. Texto \& Contexto Enfermagem. 2009; 18 (2): 249-57. 12. Facchini LA, Piccini RX, Tomasi E, Thumé E, Teixeira VA, Silveira DS, et al. Avaliação de efetividade da Atenção Básica à Saúde em municípios da região Sul e Nordeste do Brasil: contribuições metodológicas. Cad Saúde Pública 2008; 24(Suppl 1):159-72.

13. Ministério da Saúde. Departamento de Atenção Básica. Programa Nacional de Melhoria do Acesso e da Qualidade da Atenção Básica (PMAQ); manual instrutivo. (Brasília): Ministério da Saúde, 2011. 62p.

14. Ministério da Saúde. Programa de Melhoria do Acesso e Qualidade da Atenção Básica-PMAQ-AB. Projeto para a avaliação externa e censo das unidades básicas de saúde. Brasília (BRASIL): Ministério da Saúde, 2012.

15. Pereira RL, Jeziorski LL, Facchini LA. Programa Nacional de Melhoria do Acesso e da Qualidade da Atenção Básica-PMAQ. In: Anais do 21을 Congresso de Iniciação Científica da Universidade de Pelotas, 2012, Pelotas, Brasil [Internet]. 2012 [acesso em: 09 abr 2014] Discponível em: http://www2.ufpel.edu.br/cic/2012/anais/pdf/CS/CS_00975.p df. 
16. Scalco SV, Lacerda JT, Calvo MCM. Modelo para a avaliação da gestão de recursos humanos em saúde. Cad Saúde Pública. 2010; 26(3): 603-14.

17. Ministério da Saúde. Secretaria de Atenção à Saúde. Núcleo Técnico da Política Nacional de Humanização. HumanizaSUS: Documento base para gestores e trabalhadores do SUS. Brasília, (Brasil): Ministério da Saúde, 2008. 72 p.

18. Cavalini ME, Bisson MP. Farmácia Hospitalar hum enfoque los Sistemas de Saúde. 1st ed. Barueri, São Paulo: Manole; 2002.

19. Anacleto TA, Perini E, Rosa MB, César CC. Crug-dispensing errors in the hospital pharmacy. Clinics. 2007; 62(3): 243-250. 20. Ministério da Saúde. Secretaria de Atenção à Saúde. Departamento de Atenção Básica. Manual de estrutura física das unidades básicas de saúde: saúde da família. Brasília (Brasil): Ministério da Saúde, 2008. 52 p.

21. Coelho MO, Jorge MSB, Araújo ME. O acesso por meio do acolhimento na atenção básica à saúde. Revista Baiana de Saúde Pública. [Internet]. 2009 [Acesso em 09 abr 2014]; 33(3): 440-452. Disponível em:

http://inseer.ibict.br/rbsp/index.php/rbsp/article/viewFile/225 /pdf_40.

22. Siqueira FCV, Facchini LA, Silveira DS, Piccini RX, Thumé E, Tomasi E. Barreiras arquitetônicas a idosos e portadores de deficiência física: um estudo epidemiológico da estrutura física das unidades básicas de saúde em sete estados do Brasil. Ciência \& Saúde Coletiva. 2009; 14(1):39-44, 2009.

23. Coelho MO, Jorge MSB. Tecnologia das relações como dispositivo do atendimento humanizado na atenção básica à saúde na perspectiva do acesso, do acolhimento e do vínculo. Ciência \& Saúde Coletiva. 2009; 14(Supl. 1):1523-1531.

24. Rosário SSD, Fernandes APNL, Batista FWB, Monteiro AI. Acessibilidade de crianças com deficiência aos serviços de saúde na atenção primária. Rev. Eletr. Enf. [Internet]. 2013 [Acesso em: 09 abr 2014]; 15(3):740-6. Disponível em: http://dx.doi.org/10.5216/ree.v15i3.19272.

25. França ISX, Pagliuca LMF, Baptista RS, França EG, Coura AS, Souza JA. Violência simbólica no acesso das pessoas com deficiência às unidades básicas de saúde. Rev Bras Enferm. [Internet]. 2010 [Acesso em: 09 abr 2014]; 63(6): 964-70. Disponível em: http://www.scielo.br/pdf/reben/v63n6/15.pdf.

Recebido: $11 / 04 / 2014$

Aceito: $16 / 03 / 2015$.

Publicado: 30/09/2015. 